\title{
e-Phaïstos
}

e-Phaïstos Revue d'histoire des techniques / Journal of the history of technology

VIII-2 | 2020

Aviation : émergence d'un complexe technique, 1900-1930

\section{La « Belle Époque » de l'absinthe à Fougerolles (Haute-Saône)}

The Belle Epoque of Absinthe in Fougerolles (Haute-Saône)

\section{Abdelhak El Mostain}

\section{OpenEdition}

Journals

Édition électronique

URL : https://journals.openedition.org/ephaistos/8167

DOI : 10.4000/ephaistos.8167

ISSN : 2552-0741

Éditeur

IHMC - Institut d'histoire moderne et contemporaine (UMR 8066)

Référence électronique

Abdelhak El Mostain, « La « Belle Époque » de l'absinthe à Fougerolles (Haute-Saône) », e-Phaïstos [En ligne], VIII-2 | 2020, mis en ligne le 27 octobre 2020, consulté le 17 septembre 2021. URL : http:// journals.openedition.org/ephaistos/8167 ; DOI : https://doi.org/10.4000/ephaistos.8167

Ce document a été généré automatiquement le 17 septembre 2021

Tous droits réservés 


\title{
La «Belle Époque » de l'absinthe à Fougerolles (Haute-Saône)
}

The Belle Epoque of Absinthe in Fougerolles (Haute-Saône)

\author{
Abdelhak El Mostain
}

\section{L'absinthe : entre Suisse et Franche-Comté}

1 Pour certains, «c'est en Suisse qu'elle [l'absinthe] est née et c'est la Suisse qui l'a donnée à la France » (Bonneff 1913); pour d'autres, l'absinthe est un breuvage français introduit sur le sol suisse par un médecin français échappant à la tourmente révolutionnaire de 1789 (Monin 1891). De fait, au début des années 1780, un médecin français, dénommé Ordinaire, choisit Couvet, un joli petit village situé dans le Val-deTravers, en Suisse, à 734 mètres d'altitude, comme siège de son activité médicale ${ }^{1}$. Ce " grand original de grande taille dit la tradition, chevauchant partout dans le vallon sur un petit cheval corse ${ }^{2}$ » joignait à l'exercice de la médecine celui de la pharmacie. Et parmi les remèdes naturels qu'il proposait à ses patients, il y avait l'élixir d'absinthe, composé de plantes aromatiques dont il avait le secret. Après en avoir fait usage, bien des gens « se déclarèrent guéris, et le médecin ne pouvait guère faire autrement que de s'en féliciter et d'en conseiller et prescrire l'emploi ${ }^{3}$ ».

2 À sa mort, le médecin légua sa recette à sa servante, qui la revendit. En 1797, le secret de fabrication était devenu la propriété d'un industriel suisse, Henri-Louis Perrenod, dont le patronyme modifié en "Pernod» est attaché à l'histoire de l'absinthe et de l'apéritif anisé (Monin 1891). Pressentant le succès de cette liqueur en France, HenriLouis Pernod fonda en 1805, à Pontarlier, une fabrique destinée à devenir entre les mains de ses petits-fils la maison «Pernod fils ». De son côté, l'aîné des Pernod fondait la maison Édouard Pernod, en 1827. Pontarlier devint la capitale de l'absinthe, et comptait en 1906 pas moins de 25 distilleries (Abraham 1994). D'abord boisson des artistes et des militaires, elle fut adoptée par les milieux populaires en raison de son faible prix. À la fin du siècle, l'absinthe était devenue l'apéritif le plus consommé en 
France : plus de 200000 hectolitres par an vers 1900, la consommation hors de la métropole non comprise ${ }^{4}$.

3 Spécialistes de la fabrication du Kirsch (El Mostain 2007), les distillateurs fougerollais usèrent de leur savoir-faire pour se lancer eux-aussi dans la fabrication de l'absinthe, dont ils tirèrent de substantiels profits. Si l'on en croit l'inscription de la distillerie Servain, la fabrication de l'absinthe était pratiquée à Fougerolles en 1857 (fig.1).

Fig.1. « Fabrique de kirsch et d'absinthe, le 25 mai 1857 »

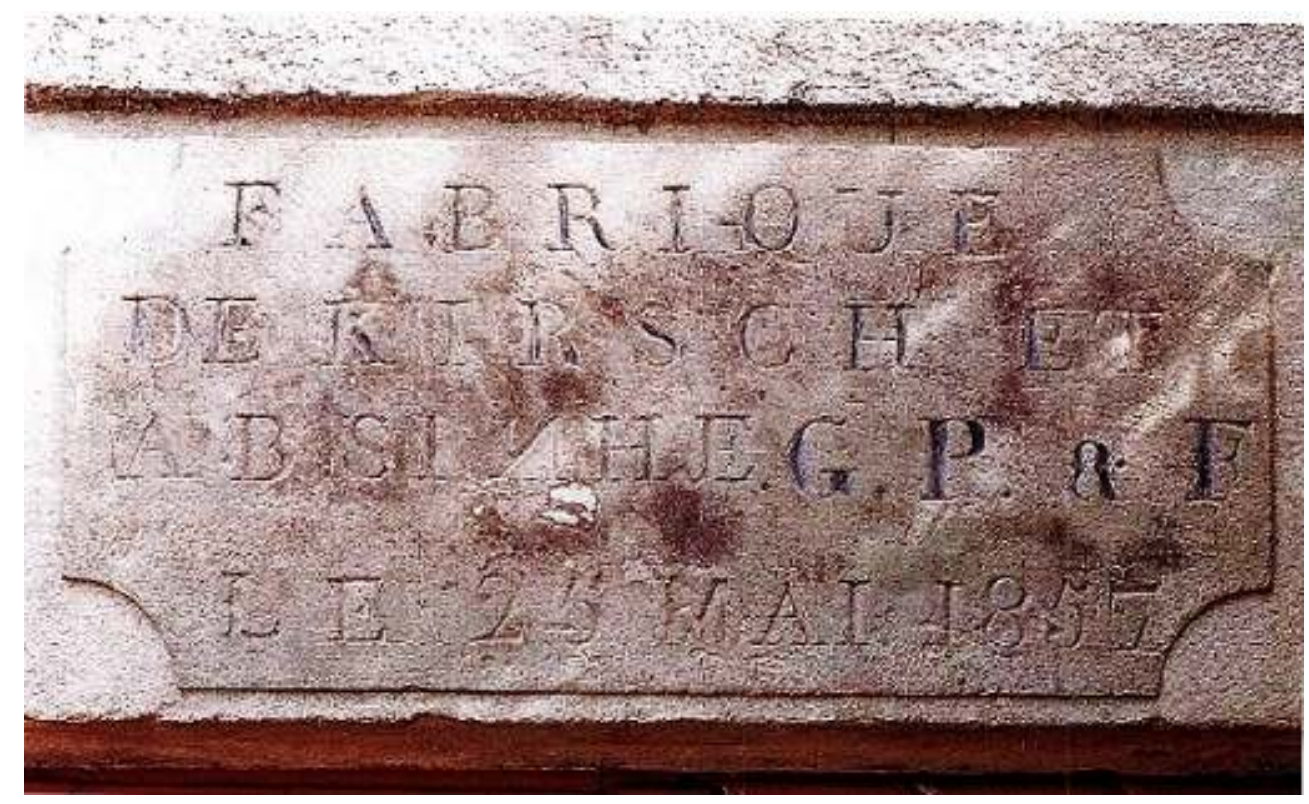

Inscription gravée en haut d'entrée de la ferme-distillerie Servain à Fougerolles

Cliché A. El Mostain

4 Avec un déficit d'image cependant : la clientèle, en effet, avait pris l'habitude au bistrot de commander non pas "une absinthe », mais « un Pernod». Abel Bresson, l'un des grands distillateurs de Fougerolles, décida, en 1912, d'acquérir l'enseigne Édouard Pernod de manière à pouvoir porter sur ses étiquettes le nom de la maison qui avait lancé l'absinthe en France (Frœmer 1884). Certains distillateurs fougerollais eurent moins de scrupules et enrôlèrent des «Pernot» locaux: Lemercier Frères commercialisa la marque "Constant Pernot», Cuenin-Rapenne, la marque "Vve J. Pernot ", les fils d'Auguste Peureux, la marque "Jules Pernot», la distillerie Louis Lemercier, la marque "Jean-François Pernot» tandis que la distillerie Saguin optait pour la marque «J-P. Pernot»! Le marché, était à ce point dynamique, que chacun y trouva sa place... En 1910, la production d'absinthe s'élevait à $23800 \mathrm{hl}$, pour les « trois grands » fougerollais, l'établissement Abel Bresson (13 124hl), Lemercier Frères (6 452hl), Auguste Peureux (4 225hl). Quatre ans plus tard, en 1914, la production globale fougerollaise atteignait les $55000 \mathrm{hl}$.

\section{Quand le malheur du vin fait le bonheur de l'apéritif}

5 L'engouement pour ce breuvage insolite s'était accéléré avec la crise du phylloxéra, qui commença à toucher les vignobles à la fin des années 1860. Le vin était le produit leader sur le marché des alcools de bouche, et il ne souffrait d'aucune concurrence notable, du 
moins jusqu'au début des années 1870. La crise du phylloxera obligea les viticulteurs à procéder à l'arrachage de leurs vignes; la production de vin ne cessa de baisser jusqu'à l'étiage, au début des années 1890. Le plus bas niveau de production fut atteint en effet en 1889 avec 23,2 millions d'hectolitres produits ${ }^{5}$. En regard, l'augmentation assez générale du niveau de vie, l'impact d'une publicité omniprésente et alléchante, la croissance du nombre des débits de boissons ( $+32 \%$ entre 1869 et 1913) et celle du nombre de bouilleurs de cru $(+1078 \% \text { entre ces deux dates })^{6}$, drainèrent une nouvelle clientèle vers les apéritifs.

De surcroit, les fabricants d'absinthe, qui avaient besoin d'alcool pour sa fabrication, durent remplacer l'alcool de vin par l'alcool industriel, issu principalement de la distillation des betteraves. Il en résulta une réduction du coût de fabrication. En 1880, un verre d'absinthe coûtait 10 centimes contre 20 centimes pour un verre de vin ${ }^{7}$. L'absinthe devint l'apéritif le plus consommé à la fin du XIX siècle. "Du nord au Midi, des Alpes à l'Atlantique, l'absinthe est la reine ", observait le quotidien Le Temps en $1884^{8}$. En 1900, il s'est bu $238000 \mathrm{hl}$ d'absinthe $(0,621 / \mathrm{hab}$.) contre $18000 \mathrm{hl}(0,0491 /$ hab.) en 1880 (Jacquet 1912). Un pareil engouement profita aux fabricants, aux distributeurs, mais aussi à l'État qui, en 1914, perçut 50 millions de francs de droits et taxes sur le produit.

\section{La chute de la « fée verte »}

7 Le succès de l'absinthe précipita sa chute. Irrités par sa popularité, les mouvements de tempérance et les lobbies viticoles se lancèrent dans une campagne de dénigrement. La «fée verte» devint le "péril vert » et la lutte pour interdire ce "poison social», un combat pour délivrer la nation. Elle fut tantôt associée à la folie ${ }^{9}:$ « Si l'absinthe n'est pas interdite, notre pays deviendra rapidement un vaste cabanon où une moitié des Français sera occupée à passer des camisoles de force à l'autre ", écrit un observateur en 1890 (Granier 1890); tantôt à la mort, tel un certain Alcyone en 1896, dans le mensuel L'alcool : 


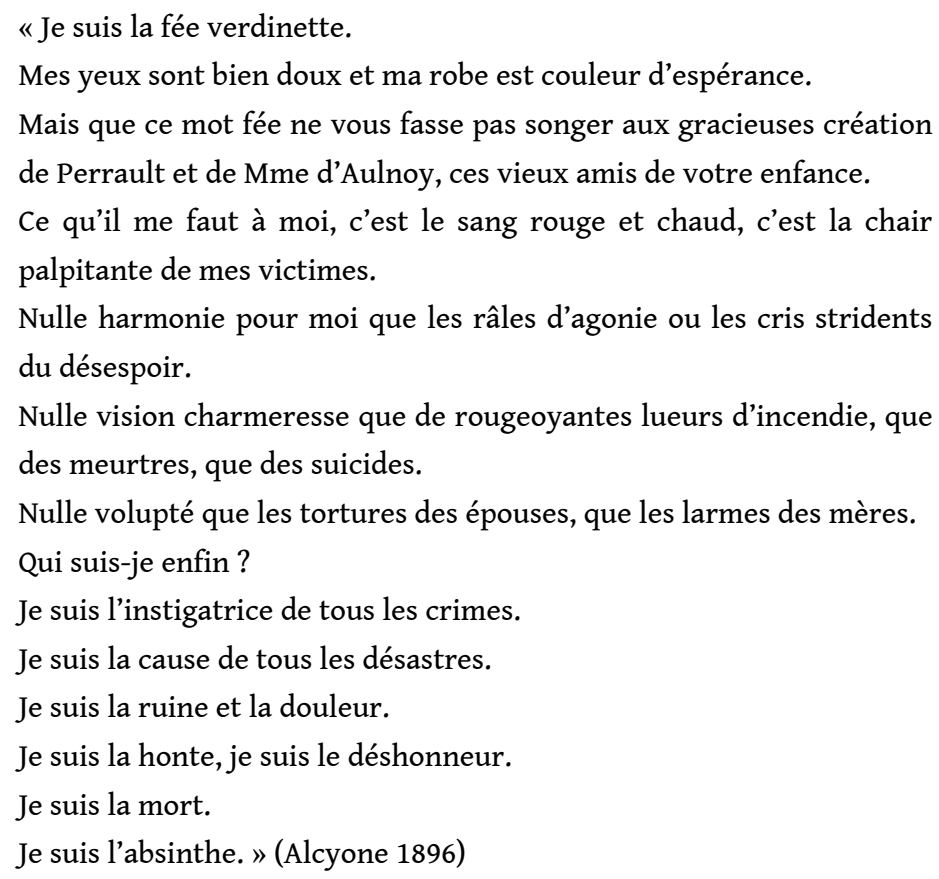

8 Malgré ces attaques, toutes les propositions de lois tendant à interdire sa consommation furent rejetées. L'engouement des Français pour cette boisson ne se démentit pas, amplifié par les stratégies de communication des fabricants et des distributeurs, qui s'organisèrent. Ainsi les distillateurs créèrent en 1892, à Fougerolles, le syndicat des distillateurs haute-saônois, et certains se firent élire, tel Auguste Peureux, qui exerça son mandat de député de la Haute-Saône à la chambre des députés du 27 avril 1902 au 31 mai 1906.

9 Le vent tourna à la fin de l'année 1914. Après avoir demandé aux préfets de prendre des arrêtés interdisant sa vente dans les débits de boissons, le gouvernement publia en janvier 1915 un décret interdisant la circulation et la vente en gros et en détail de l'absinthe et des liqueurs anisées. En mars 1915, le décret se transformait en loi ${ }^{10}$. La disgrâce dura jusqu'à la fin du XX ${ }^{\mathrm{e}}$ siècle. En 1988 en effet, un décret autorisait et règlementait la présence de thuyone dans les boissons et l'alimentation, ce qui permit techniquement de produire à nouveau de l'absinthe en France, mais uniquement sous l'appellation «spiritueux à base de plantes d'absinthe " ${ }^{11}$. La production reprit en 1999. En 2011, l'appellation "absinthe " fut remise en vigueur pour contrer une tentative d'appropriation par des producteurs suisses ${ }^{12}$. En 2013 enfin, «l'absinthe de Pontarlier » bénéficiait de l'appellation IGP (Indication Géographique Protégée).

\section{Un apéritif, une foule de recettes}

10 La grande absinthe, la petite absinthe, l'anis, le fenouil et l'hysope forment la base de cette boisson. Mais plusieurs autres produits tels l'anis étoilé, la mélisse, la menthe, l'ortie, la coriandre, l'iris ou le benjoin, peuvent être rajoutés, et les fabricants ne s'en privaient pas. En fait, il existait une foule de recettes, selon les régions et les fabricants. Chaque producteur possédait sa recette ou plutôt ses recettes. Les fabricants d'absinthe les modifiaient en fonction des différentes marques qu'ils commercialisaient et du goût 
de leur clientèle. Ils positionnaient commercialement leurs produits par rapport à la quantité et la qualité des plantes utilisées.

Fig.1. Tableau comparatif de quelques recettes d'absinthe fabriquées en France

\begin{tabular}{|c|c|c|c|c|c|c|}
\hline $\begin{array}{c}\text { Process } \\
\text { de fabrication }\end{array}$ & Ingrédients & Fougerolles & Pontarlier & Neuchâtel & Lyon & Montpellier \\
\hline \multirow{6}{*}{$\begin{array}{l}\text { Macération } \\
\text { et distillation }\end{array}$} & Grande absinthe & $2,75 \mathrm{~kg}$ & $2,5 \mathrm{~kg}$ & $6 \mathrm{~kg}$ & $3 \mathrm{~kg}$ & $2,5 \mathrm{~kg}$ \\
\hline & Anis vert & $7,5 \mathrm{~kg}$ & $5 \mathrm{~kg}$ & $6 \mathrm{~kg}$ & $8 \mathrm{~kg}$ & $6 \mathrm{~kg}$ \\
\hline & Fenouil & $4 \mathrm{~kg}$ & $5 \mathrm{~kg}$ & $5 \mathrm{~kg}$ & $4 \mathrm{~kg}$ & $4 \mathrm{~kg}$ \\
\hline & Coriandre & & & $1 \mathrm{~kg}$ & & $1 \mathrm{~kg}$ \\
\hline & Angélique (semences) & & & & $0,5 \mathrm{~kg}$ & $0,5 \mathrm{~kg}$ \\
\hline & Alcool $85^{\circ}$ & 95 litres & 95 litres & & 95 litres & 95 litres \\
\hline \multirow{5}{*}{ Coloration } & Hysope & $600 \mathrm{~g}$ & $1 \mathrm{~kg}$ & $1 \mathrm{~kg}$ & $500 \mathrm{~g}$ & $750 \mathrm{~g}$ \\
\hline & Mélisse citronné & $750 \mathrm{~g}$ & $500 \mathrm{~g}$ & $1 \mathrm{~kg}$ & $1 \mathrm{~kg}$ & $750 \mathrm{~g}$ \\
\hline & Menthe poivrée & $600 \mathrm{~g}$ & & $1 \mathrm{~kg}$ & & \\
\hline & Petite absinthe & $700 \mathrm{~g}$ & $1 \mathrm{~kg}$ & & $1 \mathrm{~kg}$ & $1 \mathrm{~kg}$ \\
\hline & $\begin{array}{l}\text { Réglisse des bois } \\
\text { Véronique }\end{array}$ & & & $\begin{array}{c}500 \mathrm{~g} \\
1 \mathrm{~kg}\end{array}$ & $500 \mathrm{~g}$ & \\
\hline
\end{tabular}

Source: Archives de l'écomusée du pays de la cerise, Fougerolles et d'après J. Fritsch, Nouveau Traité de la fabrication des liqueurs, Paris, 1891.

11 À Fougerolles, on employait l'anis, la badiane, le fenouil, l'hysope, la petite absinthe, la coriandre, la mélisse, la menthe et l'ortie. La grande absinthe n'entrait que pour un sixième environ dans le mélange global. La teneur en essences (de 1 à $4 \mathrm{gr} / \mathrm{l}$ ) était généralement proportionnelle à la force alcoolique du spiritueux. Autour des trois grandes sortes d'absinthes: l'absinthe supérieure $\left(65\right.$ à $\left.72^{\circ}\right) ; l^{\prime}$ 'absinthe ordinaire (50 à $\left.65^{\circ}\right)$; et l'absinthe commune ( 40 à $\left.50^{\circ}\right)$, on trouvait une gamme impressionnante de produits. La maison Abel Bresson, par exemple, commercialisait dix absinthes différentes en 1914, avec une communication commerciale centrée sur quatre marques nationales: l'absinthe Abel Bresson, le produit haut-de-gamme; l'absinthe Pierrot; l'absinthe des artistes moyenne gamme; l'absinthe Primia, une marque issue d'une collaboration avec plusieurs distilleries françaises réservée au marché parisien; et l'absinthe internationale Édouard Pernod, acquise en 1912 (fig.2). 
Fig. 2. Marques d'absinthe appartenant à l'Établissement Abel Bresson entre 1842 et 1915
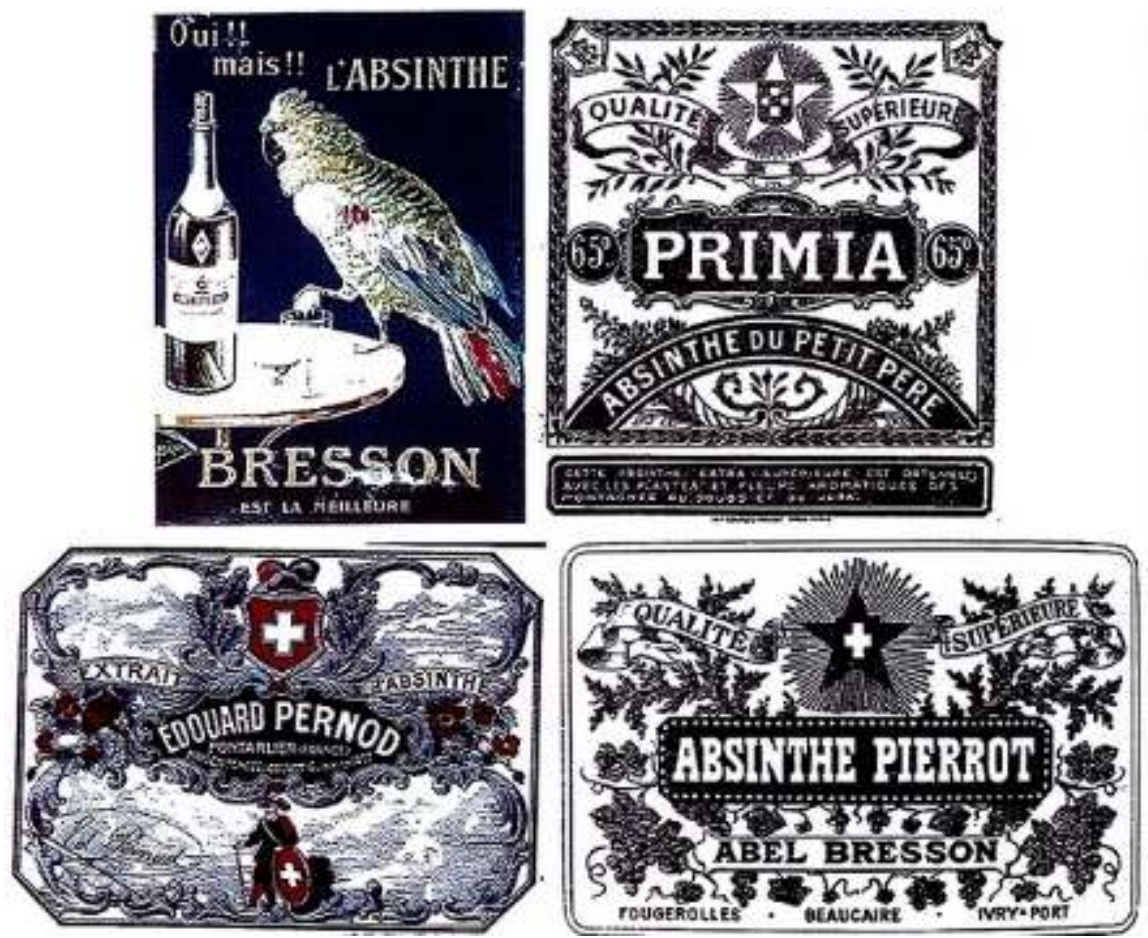

Source : Écomusée du pays de la cerise, Fougerolles.

\section{La maîtrise du complexe technique de production}

Pour endiguer la concurrence et maintenir leurs parts de marché, les industriels travaillèrent à maîtriser la plus grande partie du complexe technique de production, des approvisionnements jusqu'à la commercialisation des produits finis, de la sélection des matières premières jusqu'au conditionnement des absinthes en passant par la macération, la distillation, la coloration et le vieillissement.

\section{Le choix des plantes}

Les distillateurs fougerollais avaient le choix entre deux sortes de plantes: l'absinthe dite de Pontarlier, qui se récoltait aux environs de Pontarlier et dans la région suisse de Val-de-Travers, et l'absinthe dite de Paris qui se récoltait aux environs de Paris (Fritsch 1891). Les distillateurs fougerollais optèrent pour l'absinthe cultivée dans les environs de Pontarlier et dans la partie haute de l'arrondissement de Besançon, parce que le sol, le climat, l'altitude de ces régions donnaient à la plante une finesse et un arôme de qualité supérieure (AFAS 1893 : 263) (fig.3). Vers 1900, la culture de la grande absinthe couvrait 1500 ha dans le Doubs ${ }^{13}$. Quelques distillateurs fougerollais, parmi eux Auguste Peureux au début du $\mathrm{XX}^{\mathrm{e}}$ siècle, tentèrent de cultiver la feuille d'absinthe à Fougerolles, sans succès, car le terroir local ne le permettait pas. 
Fig.3. Récolte de l'absinthe dans la région de Pontarlier, fin $\mathrm{XIX}^{\mathrm{e}}$-début $\mathrm{XX}$ siècle

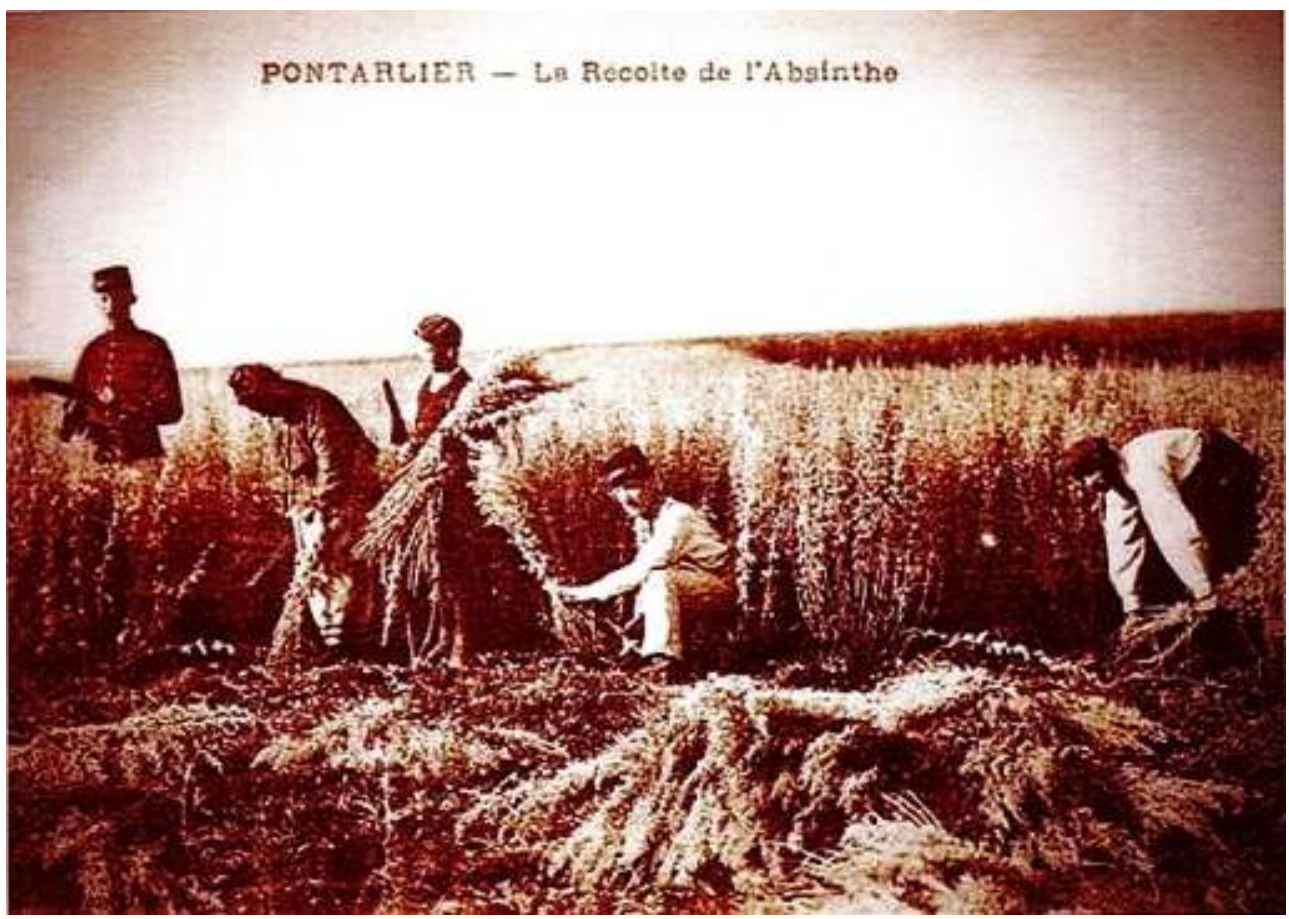

Source : Collection privée

Les plantes complémentaires provenaient d'autres régions de France, voire de l'étranger. Le fenouil venait principalement du Tarn et du Gard ${ }^{14}$; l'hysope du Doubs. Quant à l'anis, il venait de Tours et d'Albi pour la liqueur de qualité supérieure, d'Alicante ou celui de Russie, pour l'absinthe commune.

\section{Rectifier l'alcool de distillation}

Les moûts fermentés, obtenus le plus souvent avec de la betterave, devaient être transformés pour en extraire l'alcool. Une première opération donnait des alcools bruts assez impurs, appelés "flegmes ", que l'on concentrait et raffinait lors d'une seconde opération, la « rectification». La rectification donnait un alcool suffisamment neutre pour être consommé directement après réduction du degré alcoolique par adjonction d'eau pure, ou être utilisé comme alcool de macération. La maison Abel Bresson fut la première à ouvrir une usine de production d'alcool industriel à base de betteraves à la fin des années 1850. L'établissement Lemercier frères fit de même au plus fort du marché, en 1895 15(fig.4), bientôt suivi par la maison Auguste Peureux en 1899. 
Fig.4. En-tête de facture des établissements Lemercier frères, datant de 1908

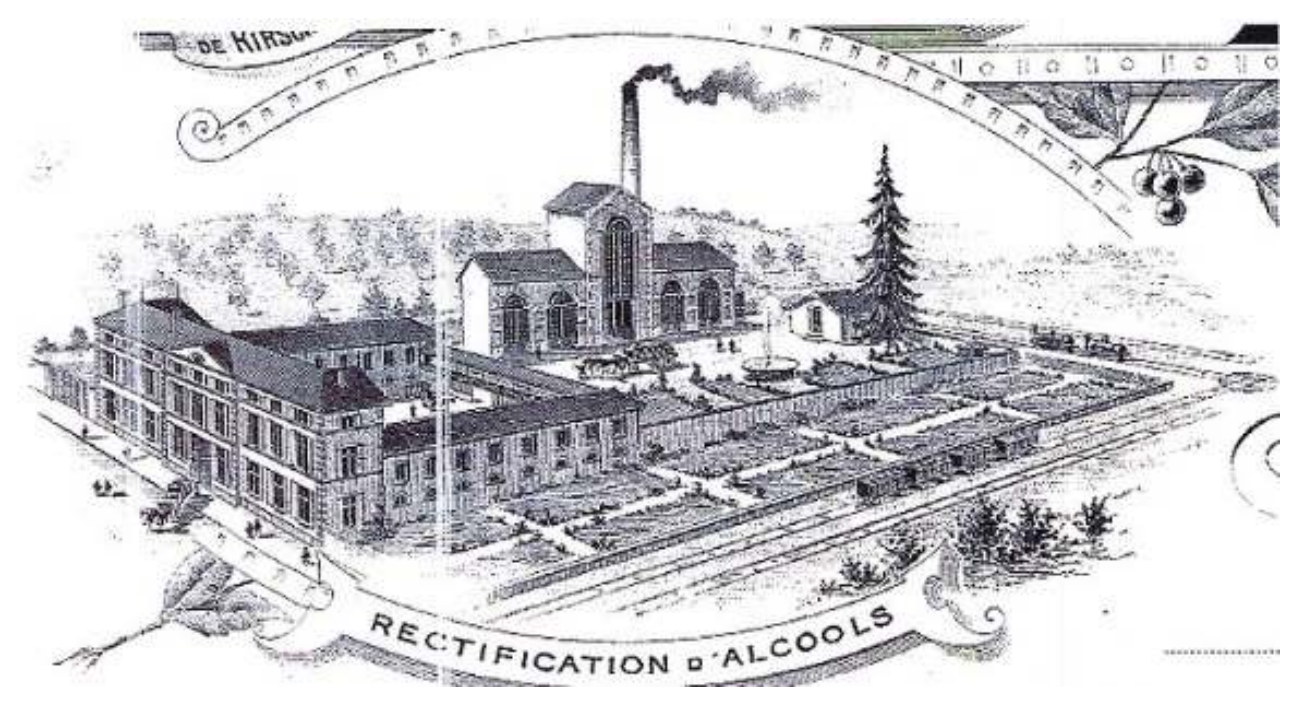

Source : Écomusée du pays de la cerise, Fougerolles

Pour l'installation de l'usine, l'établissement Abel Bresson fit appel à deux constructeurs industriels déjà leaders sur le marché national: Savalle et Egrot, et fit installer une machine à vapeur pour actionner les divers mécanismes de l'usine, comme la pompe à eau et le broyeur. Un générateur, installé dans la chaufferie, fournissait la vapeur nécessaire à la machine et aux appareils de distillation.

On utilisait à Fougerolles le procédé dit de « distillation continue en colonne » (Jacquet 1912), une technique nouvelle qui s'adaptait à toutes les substances à distiller: les fruits, les mélasses, les graines... mais surtout à la betterave. L'alcool obtenu était soit utilisé comme produit intermédiaire, y compris chez les contrefacteurs (voir infra), soit vendu comme alcool de bouche, sous l'appellation «Le chien ». La distillation (fig.5) s'effectuait par un système d'extraction à contre-courant qui maximisait l'efficacité de la concentration en alcool dans les distillats (Jacquet 1912). Dans leur mouvement ascendant, les vapeurs s'enrichissaient en alcool, tandis que, dans le mouvement inverse, les moûts s'épuisaient. Plus il y avait de plateaux, plus le degré d'alcool obtenu était élevé ; on pouvait passer ainsi de $55^{\circ}$ à plus de $90^{\circ}$ d'alcool pur. La vapeur était ensuite refroidie à travers un condenseur alimenté régulièrement en eau. 
Fig.5. Principe de fonctionnement de la colonne de rectification

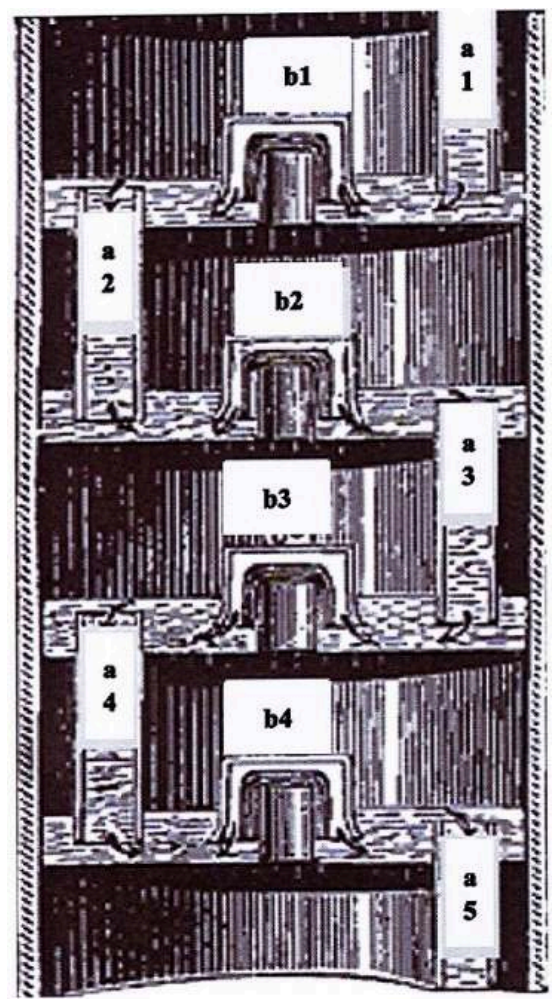

Une série de plateaux superposés forme autant de petits appareils de distillation distincts. Le liquide à distiller arrive en a1, par la partie supérieure, descend, de plateau en plateau, en a2, a3, a4, a5, par des tuyaux de trop-plein, jusqu'à la partie inférieure, où il arrive épuisé pour être expulsé au-dehors sous forme de vinasses. La vapeur suit une marche inverse et, grâce à des calottes b1, b2, b3, b4, disposées convenablement à cet effet, sur les plateaux, est obligée, pour s'échapper, de barboter à travers le liquide. Les vapeurs dans leur mouvement ascendant, s'enrichissent en alcool, tandis que, dans le mouvement inverse, les moûts s'épuisent.

Source : Jacquet, L'alcool, étude économique générale, Masson, Paris, 1912, p. 141.

\section{Une même chaîne opératoire}

Quelques divers que pouvaient être les ingrédients, le procédé de fabrication demeurait le même, avec quatre phases successives : la macération, la distillation, la coloration et le vieillissement.

\section{La macération des plantes dans l'alcool rectifié, une étape capitale}

19 Les plantes étaient sélectionnées et préparées. Pour l'absinthe de qualité supérieure, on effeuillait l'absinthe et l'hysope, en évitant le bois, moins aromatique que les feuilles et les fleurs, le procédé de macération pouvait débuter. On les dosait et on les mettait dans l'alambic; on chargeait la quantité voulue d'alcool, à quoi on ajoutait seulement la moitié de la quantité d'eau nécessaire pour la distillation, une précaution nécessaire à la réussite de la macération. Ajouter toute la quantité d'eau avait pour effet de trop diluer l'alcool. A l'inverse, macérer dans l'alcool pur provoquait un racornissement des plantes ce qui nuisait au développement de leur arôme. La macération durait de 12 à 24 heures. 


\section{La distillation en alambic}

20 plantes devait être conduite avec lenteur et grand soin. On recueillait l'esprit de l'alcoolat parfumé qui marquait 70 à $72^{\circ}$. Appelées flegmes d'absinthe, la tête (les flegmes coulant au début de la distillation) et la queue (les flegmes de moins de $60^{\circ}$ coulant à la fin de la distillation) servaient à la fabrication des absinthes ordinaires. Le cœur de distillation donnait l'extrait d'absinthe proprement dite. Quant au produit blanchâtre et laiteux qui coulait en toute fin, encore riche en essence, il était versé dans la distillation suivante avec l'alcool et les plantes.

Dans la plupart des distilleries fougerollaises, les alambics de 150 à 300 litres à feu nu ou à bain-marie s'effacèrent progressivement dans le dernier tiers du $\mathrm{XIX}^{\mathrm{e}}$ siècle (El Mostain 2017), pour laisser place à des alambics à vapeur perfectionnés de 600 à 1200 litres (Frœmer 1884) (fig.6).

Fig.6. Établissement Abel Bresson, alambics à vapeur jumelés pour la fabrication de l'absinthe début $\mathrm{XX}$ siècle

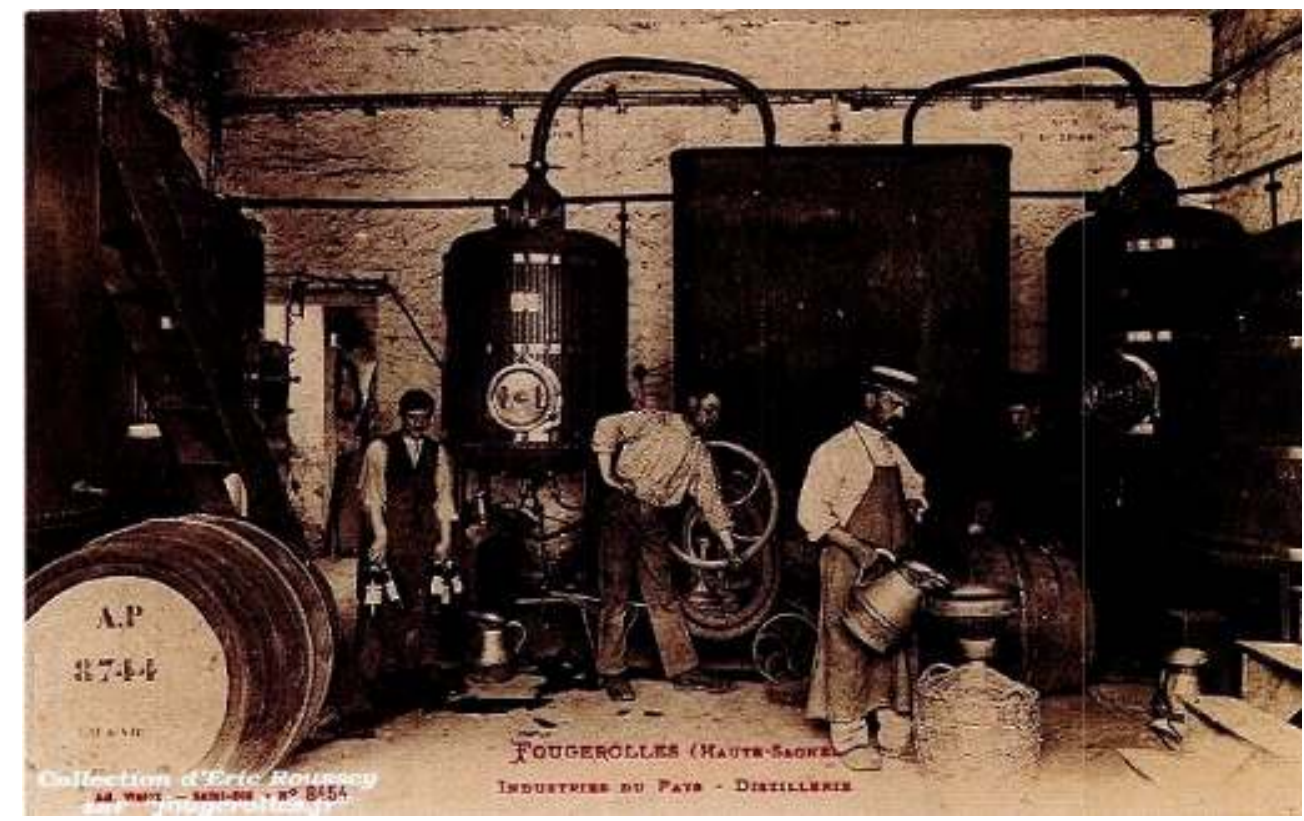

Source : Collection Marcel Saire

\section{La coloration et le vieillissement, deux paramètres de qualité et de notoriété}

Parfaitement limpide comme tout liquide distillé soigneusement, l'extrait d'absinthe était coloré pour le conformer au marché des alcools de bouche en France. Les petits producteurs fougerollais utilisaient pour ce faire le même alambic de distillation, entre deux opérations. Chez les distillateurs industriels, Abel Bresson, Lemercier frères et Auguste Peureux, la coloration s'effectuait dans un autre alambic, à double fond en cuivre étamé, chauffé à la vapeur ou par circulation d'eau chaude et muni d'un couvercle portant un tuyau en communication avec un serpentin cohobateur, appelé 
«colorateur ». En 1878, l'établissement Abel Bresson en possédait deux pour les infusions d'absinthe ${ }^{16}$.

Les substances colorantes étaient choisies en fonction de la qualité d'absinthe à atteindre ; par exemple, plus on met de petite absinthe, plus la couleur tire sur le noir ; plus on force la proportion d'hysope, plus on se rapproche de la teinte feuille morte (Fritsch 1891). À Fougerolles, en plus de la petite absinthe et de l'hysope, les distillateurs incorporaient de la mélisse citronnée et de la menthe poivrée ${ }^{17}$. L'opération durait de 12 à 24 heures. Pour une question économique, les plantes servant à la coloration de l'absinthe n'étaient pas complètement épuisées après la première opération; les distillateurs les rechargeaient avec l'absinthe de qualité ordinaire, qui achevait de les épuiser. Il en résultait une coloration plus faible, qu'on renforçait par une addition de caramel.

Après refroidissement, le liquide coloré était mis à vieillir pendant 2 à 5 ans pour compléter la qualité. L'absinthe vieillissait dans des foudres d'une contenance moyenne de 1200 litres chez les industriels de la distillation et dans des fûts chez les petites distilleries (fig.7). Pour accélérer la commercialisation des absinthes fabriquées, plusieurs fabricants eurent recours à l'usage de l'ozone issu de l'électrisation de l'oxygène pur (Villon 1902), dont ils firent un argument commercial, ainsi l'« Absinthe ozonisée Lemercier frères ${ }^{18}$ » ou l'«Absinthe oxygéné Abel Bresson ${ }^{19}$ ». Suivant les qualités de la liqueur, on se servait d'oxygène pur, d'oxygène électrisé ou alternativement, d'un mélange des deux. L'absorption de 25 litres d'oxygène par hectolitre d'absinthe suffisait pour atteindre le résultat recherché.

Fig.7. Lemercier frères, foudres accueillant l'absinthe à vieillir avant son expédition

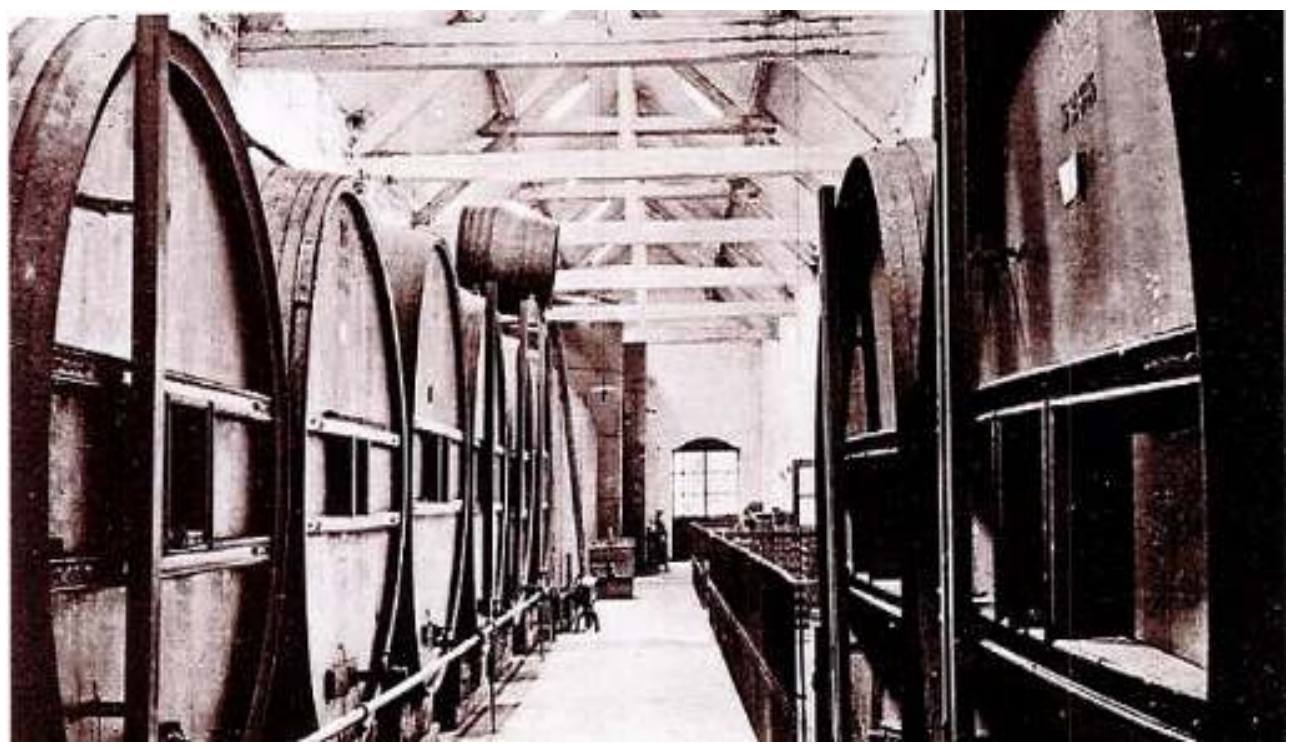

Ces foudres serviront plus tard pour les alcools anisés héritiers de l'absinthe après son interdiction. Source : Archives de l'Établissement Lemercier frères, Fougerolles.

À la fin du processus de vieillissement, une petite partie de la production était conditionnée dans les bouteilles typiques de l'absinthe en verre foncé ou vert, pour atténuer l'influence de la lumière. Le reste était conditionné en fûts de chêne, ce qui réduisait le coût du transport (100 litres en fûts pèsent moins lourd que 100 bouteilles en verre, Guy 1993) et autorisait des marges plus importantes. Puis, eu égard à 
l'accroissement considérable de la demande, ils affrétèrent leurs propres wagonsciternes (fig. 8). À partir de 1881, année d'ouverture de la gare ferroviaire de Fougerolles (Guy 1977), les distilleries se rapprochèrent de la voie ferrée. En 1892, l'établissement Lemercier frères conclut un contrat avec la compagnie ferroviaire pour la construction d'un embranchement reliant directement la distillerie à l'entrepôt de marchandises de la gare et, en 1898, il négociait le raccordement de l'usine de rectification d'alcool ${ }^{20}$.

Fig.8. Embranchement de l'usine de distillation Lemercier Frères à la ligne de chemin de fer en 1898

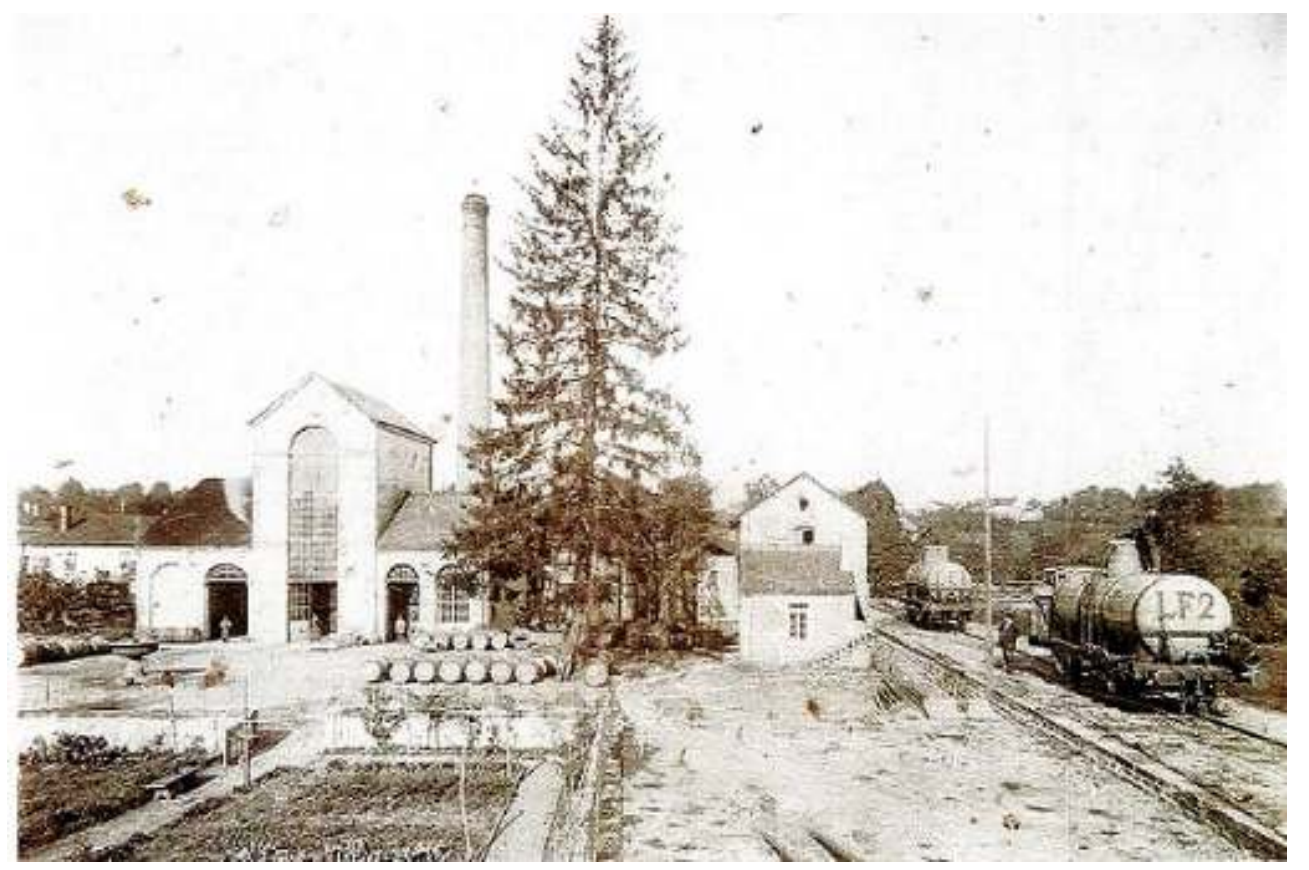

Les wagons-citernes portant le sigle LF de la distillerie.

Source : Archives de l'établissement Lemercier frères, Fougerolles.

\section{L'absinthe contrefaite}

Certains distillateurs désireux de tirer profit de ce marché rentable n'hésitèrent pas à préparer une absinthe bon marché par la simple dissolution d'essences dans l'alcool ${ }^{21}$. Ce procédé qui ne nécessitait pas de grands investissements techniques (Villon 1902), consistait à additionner directement une quantité déterminée d'essences variées dans l'alcool acquis chez les rectificateurs fougerollais.

Les distillateurs industriels dénoncèrent cette pratique, au nom de la concurrence déloyale et des arguments que cela donnait aux détracteurs de l'absinthe, les hygiénistes, les lobbies antialcooliques et les lobbies viticoles. L'établissement Abel Bresson, un des leaders sur le marché des apéritifs et digestifs en France, n'hésita pas à faire intervenir la presse. En 1884, le journaliste A. Frœmer brossait dans Le Panthéon de l'industrie, un portrait flatteur d'Abel Bresson, et opposait les " produits sérieusement hygiéniques » (Frœmer 1884) proposés par l'entreprise, aux produits obtenus par la contrefaçon, la fraude et l'imitation certes ingénieuse mais « presque toujours funeste 
pour la santé publique ». Sept ans plus tard, Lemercier Frères en faisait de même dans la même revue ${ }^{22}$.

\section{Le rituel de l'absinthe}

L'absinthe ne se prépare pas comme un apéritif classique. Elle nécessite un rituel impliquant patience et savoir-faire, accompli avec des accessoires spécifiques : la carafe ou la fontaine à absinthe (Rousseau-Aicardi 2008) (fig.9) ainsi que le verre et la cuillère à absinthe immortalisés par de nombreux artistes peintres ${ }^{23}$ dont Picasso.

Fig.9. Fontaine à absinthe avec verres et cuillère (non daté)

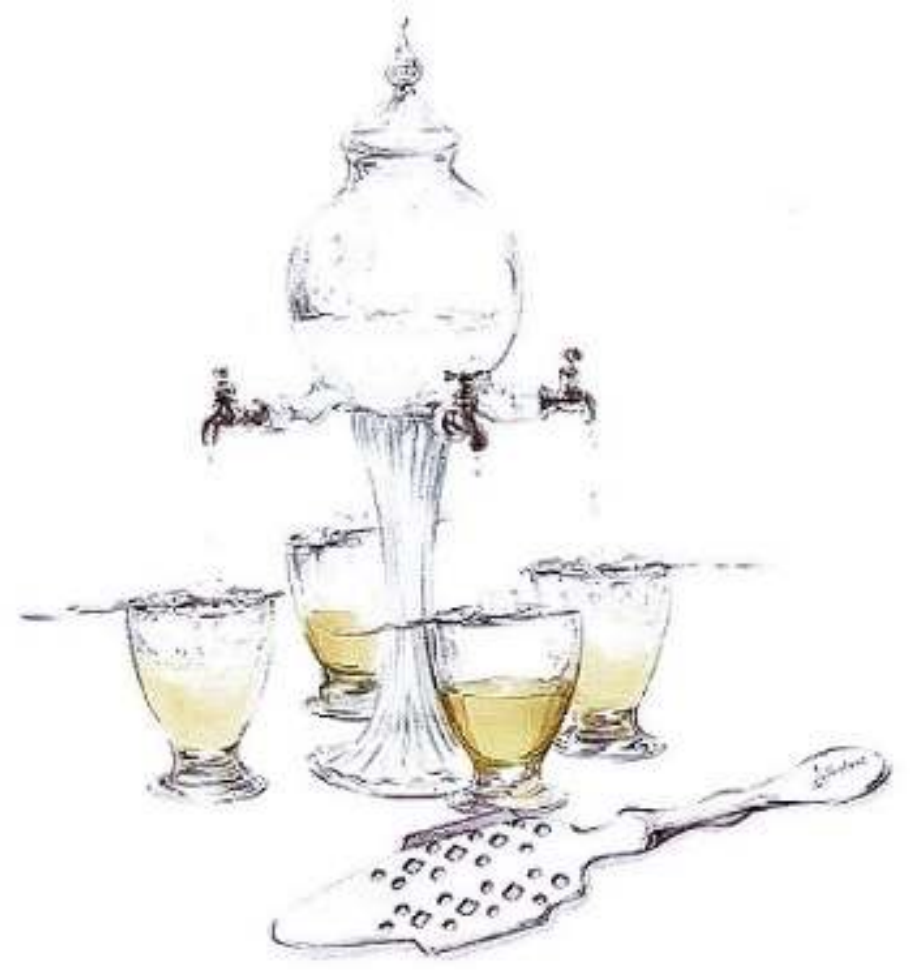

Source : Distillerie Paul Devoille, Fougerolles

On doit à Marcel Pagnol une description très évocatrice de ce rituel, telle qu'on le pratiquait à la Belle Époque :

«L'œil du poète brilla tout à coup. Alors, dans un profond silence, commença une sorte de cérémonie. Il installa devant lui un verre [...]. Il prit ensuite la bouteille, la déboucha, la flaira, et versa un liquide ambré à reflets verts, dont il parut mesurer la dose avec une attention soupçonneuse [...]. Il prit alors sur le plateau une sorte de petite pelle en argent, qui était étroite et longue, et percée de découpures en forme d'arabesques. Il posa cet appareil, comme un pont, sur les bords du verre, et le chargea, de deux morceaux de sucre [...]. L'Infante souleva la cruche [...] elle fit tomber un très mince filet d'eau fraîche [...] sur les morceaux de sucre, qui commencèrent à se désagréger lentement [...]. Dans le liquide, dont le niveau montait 
lentement, je vis se former une sorte de brume laiteuse, en torsades tournantes qui finirent par se rejoindre, tandis qu'une odeur pénétrante d'anis, rafraîchissait délicieusement mes narines " (Pagnol 2014).

Un habitant fougerollais, un certain Paul Simon, lui fait écho, avec une autre chute :

«Versez avec lenteur l'absinthe dans le verre; deux doigts, pas davantage; ensuite saisissez une carafe d'eau bien fraîche; puis versez, versez tout doucement d'une main légère. Quand vous aurez jugé la liqueur assez claire, laissez-la reposer une minute encore; couvrez-la d'un regard comme on couvre un trésor. Aspirez son parfum qui donne le bien-être! Enfin, pour couronner tant de soins inouïs, bien délicatement, prenez le verre... et puis, lancez sans hésitation le tout par la fenêtre ! ${ }^{24}$.

31 L'interdiction de « l'atroce sorcière verte » comme la qualifiait Verlaine sonne le début d'une politique antialcoolique qui se poursuit au-delà de la Grande Guerre. Cela favorisa les boissons issues de la fermentation, notamment le vin. Mais cela permit aussi l'émergence des liqueurs anisées, héritières directes de l'absinthe. Les distillateurs haut-saônois, Bresson, Lemercier et les autres, mirent à profit leur savoir-faire pour se lancer dans les apéritifs anisés ${ }^{25}$. Le rituel du pastis remplaça celui de l'absinthe, pour la plus grande fortune des distillateurs.

\section{BIBLIOGRAPHIE}

ABRAHAM Nicolas, L'immigration à Pontarlier dans l'entre-deux-guerres, mémoire de maîtrise (dir. Janine Ponty), Université de Franche-Comté, 1994

ALCYONE, L'Alcool, journal mensuel de la société contre l'usage des boissons spiritueuses, $\mathrm{n}^{\circ} 3,20$ mars 1896

Association française pour l'avancement des sciences (AFAS), Notices historiques, scientifiques et économiques, Besançon, Août 1893

BONNEFF Léon, Marchands de folie, Paris, Marcel Rivière, 1913

EL MOSTAIN Abdelhak, « Distiller les cerises à Fougerolles aux XVIII ${ }^{\mathrm{e}}$ et XIX ${ }^{\mathrm{e}}$ siècles : une petite histoire du kirsch », e-Phaïstos [En ligne], VI-1 2017 | 2018

FRITSCH Jean, Nouveau Traité de la fabrication des liqueurs d'après les procédés les plus récents, Paris, 1891

FROEMER A, « M. Abel Bresson », Le panthéon de l'industrie, n497, Paris, 7 septembre 1884

GRANIER Paul, La folie à Paris, Paris, J-B Baillière et fils, 1890 
GUY J. Michel, « L'établissement des lignes de chemins de fer en Haute-Saône », Bulletin de la Société d'Agriculture, Lettres, Sciences et Arts (SALSA), Vesoul, 1977

GUY J. Michel, « La distillation du kirsch à Fougerolles », Bulletin de la Société d'Agriculture, Lettres, Sciences et Arts (SALSA) de la Haute-Saône, n²5, Vesoul, 1993

JACQUET Louis, L'alcool, étude économique générale : ses rapports avec l'agriculture, l'industrie, le commerce, la législation, l'impôt, l'hygiène individuelle et sociale, Paris, Masson, 1912

MONIN Ernest, Dictionnaire universel de cuisine et d'hygiène alimentaire, Paris, Les libraires, 1891

PAGNOL Marcel, Le temps des secrets, Souvenirs d'enfance, Tome III, Paris, Éditions de Fallois, 2014

PIVATY Jean Jacques, Distillation et Distilleries, édition Jean Jacques Pivaty, 2011

ROUSSEAU-AICARDI Danièle, « Musée de Montmartre », Bulletin de la Société d'histoire et

d'archéologie Le Vieux Montmartre, Paris, décembre 2008

VILLON A.-Mathieu, Dictionnaire de chimie industrielle, Paris, 1902

\section{NOTES}

1. Le conteur vaudois, journal de la Suisse Romande, vingt-huitième année, $n^{\circ} 2$, samedi 11 janvier 1890.

2. La Petite presse, Paris, 25 août 1874.

3. Ibidem.

4. Archives Départementales de la Haute-Saône (ADHS), 106 M1, Fougerolles, 30 décembre 1895 : La veuve Bresson produit en $189518573 \mathrm{hl}$ d'alcool et en vend $22133 \mathrm{hl}$ (soit respectivement $3588 \mathrm{hl}$ et $1283 \mathrm{hl}$ de plus qu'en 1894). Elle explique que "cette augmentation porte sur la vente de l'absinthe qui s'écoule plus facilement par suite de l'institution d'un entrepôt à Alger et dans divers centres de la France ».

5. Ministère des Finances, Bulletin de statistiques de la France, 1951, p.34.

6. Bulletin de Statistiques et de Législation comparée, 1914.

7. JO Chambre, Documents parlementaires, annexes 232, 11 juillet 1911, p.927

8. Le Temps, 22 février 1894.

9. Principale molécule de l'huile essentielle d'absinthe, la tuyone est très convulsivante et susceptible de provoquer des hallucinations.

10. Décret-loi signé le 16 mars 1915 par Raymond Poincaré.

11. Avec un taux de thuyone limité à $35 \mathrm{mg} /$ litre.

12. Loi du 17 mai 2011.

13. Revue de botanique appliquée et d'agriculture coloniale, Paris, janvier 1943, p.312.

14. Rapport du Sénat $n^{\circ} 232$, session ordinaire, annexes au procès-verbal de la séance du 11 juillet 1911.

15. ADHS, 9M1. L'établissement Lemercier Frères embaucha en 1895 cinq hommes pour la rectification d'alcool qu'il venait d'installer.

16. ADHS, 2E20742. Inventaire après décès de Monsieur Abel Bresson, effectué le 16 juillet 1878.

17. ADHS, 9M1 (1894 et 1895). Données d'après un registre de commerce conservé par M. Claude Roussey (1896) et ADHS, 121J41, Registre de commerce 1900.

18. "Absinthe ozonisée Lemercier frères", marque déposée en 1908 par l'établissement Lemercier frères, Fougerolles. Archives de l'Établissement Lemercier frères, Fougerolles.

19. Archives de l'écomusée du pays de la cerise, Fougerolles. 
20. Archives de l'Établissement Lemercier Frères. Approbation par le Ministre des Travaux publics du projet d'embranchement particulier (au point kilométrique 102.327 de la ligne Aillevillers à Faymont) datant du 3 décembre 1998.

21. Le Temps, Paris, 25 mai 1895.

22. G., « Une grande distillerie à Fougerolles ", Le Panthéon de l'industrie, n 842, août 1891.

23. Edgar Degas, L'absinthe, huile sur toile 1875-1876, Musée d'Orsay, Paris ; Édouard Manet, Le buveur d'absinthe, huile sur toile 1859, Ny Carlsberg Glyptotek, Copenhague ; Pablo Picasso, La buveuse d'absinthe, huile sur carton 1901, musée d'Orsay, Paris ; Le buveur d'absinthe, huile sur toile 1903, Collection privée, Musée de l'Absinthe, Auvers-sur-Oise; Henri de Toulouse-Lautrec, Monsieur Boileau au café, peinture sur carton 1893, Cleveland Museum of Art ; Vincent Van Gogh, L'absinthe, nature morte, 1887, Musée d'Orsay, Paris.

24. Archives privées Paul Simon, Écomusée du pays de la cerise, Fougerolles, nd.

25. Matthieu Fort, «Le pastis ne viendrait pas de Marseille, mais de Haute-Saône ", d'après un entretien avec Abdelhak EL Mostain, Magazine Sparse, n²8, septembre 2019.

\section{RÉSUMÉS}

Presque un siècle après son interdiction, l'absinthe a été ré-autorisée en France par la loi du 17 mai 2011 afin de contrer une tentative de producteurs suisses de s'approprier cette appellation. Cette "fée verte ", comme l'a surnommée l'écrivain irlandais Oscar Wilde, a marqué l'histoire de la France durant la Belle Époque jusqu'en 1915, année de son interdiction. Si cette boisson est davantage connue pour être "la boisson qui rend fou», elle fut aussi et surtout un produit du terroir franco-suisse, dont Fougerolles en Haute-Saône, fut l'un des hauts lieux de fabrication avec la région de Pontarlier dans le Doubs. Si chaque fabricant possédait sa propre recette, le procédé de fabrication demeura identique : macération, distillation, coloration et vieillissement. L'engouement pour ce breuvage et sa perception dans l'imaginaire social, font de la fée verte une boisson mythique et mystique avec son rituel de consommation faisant se côtoyer, avec patience, trois accessoires : une carafe, un verre à dose et une pelle à absinthe. Au-delà de l'image funeste attribuée, à tort ou à raison, à l'absinthe, elle représente avant tout un savoir-faire et un art de consommation.

Almost a century after it was banned, absinthe is re-authorised in France by the law of 17 May 2011 in order to counter an attempt made by Swiss producers to appropriate this appellation. This "green fairy", as it was nicknamed by the Irish writer Oscar Wilde, marked the history of France during the Belle Époque until 1915. If this drink is better known as "the drink that drives you crazy", it was also and above all a product of the Franco-Swiss soil, Fougerolles in HauteSaône along with the Pontarlier region in the Doubs. Although each region and each manufacturer had its own recipes, the manufacturing process remained the same: maceration, distillation, colouring and ageing. The craze for this beverage and its perception in the social imagination make the green fairy a mythical and mystical drink with its drinking ritual that brings together, with patience, three accessories: a carafe, a measuring glass and an absinthe shovel. Beyond the fatal image attributed, rightly or wrongly, to absinthe, it represents above all, a know-how and an art of consumption. 
INDEX

Mots-clés : histoire des techniques, alcool, distillation, vin, histoire des entreprise, complexe technique, absinthe

Keywords : history of technology, alcohol, distillation, industry, absinthe, wine Thèmes : Un objet une technique

\section{AUTEUR}

\section{ABDELHAK EL MOSTAIN}

Abdelhak El Mostain est Docteur en Histoire et Civilisations, spécialité Épistémologie, Histoire des sciences et des techniques, enseignant en économie-gestion à l'IUT du Creusot, chercheur associé au laboratoire FEMTO-ST/RECITS UMR 6174, CNRS, Université Bourgogne FrancheComté/UTBM. 